\title{
Angiomotin is a novel Hippo pathway component that inhibits YAP oncoprotein
}

\author{
Bin Zhao, ${ }^{1,2,6}$ Li Li, $^{1,2,3,6}$ Qing Lu, ${ }^{1,2}$ Lloyd H. Wang, ${ }^{1,2}$ Chen-Ying Liu, ${ }^{4,5}$ Qunying Lei, ${ }^{4,5}$ \\ and Kun-Liang Guan ${ }^{1,2,7}$ \\ ${ }^{1}$ Department of Pharmacology, University of California at San Diego, La Jolla, California 92093, USA; ${ }^{2}$ Moores Cancer Center, \\ University of California at San Diego, La Jolla, California 92093, USA; ${ }^{3}$ Department of Biological Chemistry, University of \\ Michigan, Ann Arbor, Michigan 48109, USA; ${ }^{4}$ Department of Biological Chemistry, School of Medicine, Fudan University, \\ Shanghai, China 200032; ${ }^{5}$ Molecular and Cell Biology Laboratory, Institutes of Biomedical Sciences, Fudan University, Shanghai, \\ China 200032
}

The Yes-associated protein (YAP) is a transcription coactivator that plays a crucial role in organ size control by promoting cell proliferation and inhibiting apoptosis. The Hippo tumor suppressor pathway inhibits YAP through phosphorylation-induced cytoplasmic retention and degradation. Here we report a novel mechanism of YAP regulation by angiomotin (AMOT) family proteins via a direct interaction. Knockdown of AMOT family protein AMOTL2 in polarized Madin-Darby canine kidney (MDCK) cells leads to YAP activation, as indicated by decreased YAP tight junction localization, attenuated YAP phosphorylation, accumulation of nuclear YAP, and induction of YAP target gene expression. Transcriptional coactivator with PDZ-binding motif (TAZ), the YAP paralog, is also regulated by AMOT in a similar fashion. Furthermore, AMOTL2 knockdown results in loss of cell contact inhibition in a manner dependent on the functions of YAP and TAZ. Our results indicate a potential tumor-suppressing role of AMOT family proteins as components of the Hippo pathway, and demonstrate a novel mechanism of YAP and TAZ inhibition by AMOT-mediated tight junction localization. These observations provide a potential link between the Hippo pathway and cell contact inhibition.

[Keywords: YAP; angiomotin; Hippo; tight junction; cancer]

Supplemental material is available for this article.

Received June 20, 2010; revised version accepted November 5, 2010.

In multicellular organisms, the relative size of each organ is precisely controlled. In the last decade, the Yes-associated protein (YAP) transcription coactivator was shown to play an important role in organ size homeostasis, and was demonstrated to be inhibited by the Hippo signaling pathway (for review, see Zhao et al. 2010a). For example, transgenic expression of YAP in the mouse liver results in a liver three times larger than normal and leads to tumor formation if high YAP expression persists (Camargo et al. 2007; Dong et al. 2007). Similarly, in Drosophila, mutation of the Hippo pathway genes or transgenic expression of Yki, the YAP homolog, results in enlarged organ size (Kango-Singh et al. 2002; Tapon et al. 2002; Harvey et al. 2003; Jia et al. 2003; Pantalacci et al. 2003; Udan et al. 2003; Wu et al. 2003; Huang et al. 2005; Lai et al. 2005). YAP promotes cell proliferation and inhibits apoptosis by activating target transcription factors, such as the TEAD family transcription factors (Zhao et al. 2008), to induce gene expression. In Drosophila, Yki-induced genes include $c y c E$, diap1, and

\footnotetext{
${ }^{6}$ These authors contributed equally to this work.

${ }^{7}$ Corresponding author.

E-MAIL kuguan@ucsd.edu; FAX (858) 534-7628.

Article is online at http://www.genesdev.org/cgi/doi/10.1101/gad.2000111.
}

bantam microRNA (Huang et al. 2005; Nolo et al. 2006; Thompson and Cohen 2006). In mammalian cells, YAP induces Birc5 and Birc2 (Dong et al. 2007), two diap1 homologs, and many cytokines such as connective tissue growth factor (CTGF), brain-derived neurotrophic factor (BDNF), fibroblast growth factor 1 (FGF1), and amphiregulin (AREG) (Hao et al. 2008; Zhao et al. 2008; J Zhang et al. 2009 , although their contribution to organ size regulation has not been evaluated.

The YAP gene locus is amplified in human cancers, including hepatocellular carcinoma (HCC), intracranial ependymoma, oral squamous cell carcinoma, and medulloblastoma (Baldwin et al. 2005; Snijders et al. 2005; Modena et al. 2006; Zender et al. 2006; Fernandez et al. 2009). More interestingly, two reports identified YAP as a driving oncogene in the human HCC 11q22 amplicon and a mouse mammary tumor amplicon (Overholtzer et al. 2006; Zender et al. 2006). Consistently, elevated YAP expression and nuclear localization have been observed in multiple types of human cancers, including liver, colon, ovarian, lung, and prostate cancers (Zender et al. 2006; Dong et al. 2007; Zhao et al. 2007; Steinhardt et al. 2008). Recently, YAP was determined to be an independent prognostic marker for overall survival and disease-free 
survival for HCC patients (Xu et al. 2009). In cell culture, YAP could induce cellular transformation and epithelialmesenchymal transition (EMT), and is involved in cell contact inhibition, a fundamental property lost in many cancer cells that enhances their ability to invade host tissues and metastasize (Overholtzer et al. 2006; Zhao et al. 2007; Zhang et al. 2008).

Genetic and biochemical studies indicated that YAP is inhibited by the Hippo pathway (for review, see Zhao et al. 2010a). Core to the Hippo pathway is a kinase cascade in which mammalian Mst1/2 kinases (Drosophila Hippo homologs) complexed with a scaffold protein, Sav1, to phosphorylate and activate the Lats1/2 kinases (Chan et al. 2005; Callus et al. 2006). Lats1/2 are also activated by another scaffold protein, Mob1 (Hergovich et al. 2006). Lats1/2 directly phosphorylate YAP on serines in five consensus HXRXXS motifs (Zhao et al. 2007, 2010b; Hao et al. 2008). Phosphorylation of YAP S127 generates a 14-3-3-binding motif responsible for YAP cytoplasmic retention (Zhao et al. 2007). Thereby, YAP is inhibited by physical separation from nuclear-localized transcription factors and target gene promoters. Furthermore, phosphorylation by Lats1/2 also destabilizes YAP. Phosphorylation on YAP S381 primes subsequent phosphorylation by another kinase, possibly casein kinase $1(\mathrm{CK} 1 \delta / \varepsilon)$, activating a phosphodegron and causing the recruitment of $\mathrm{SCF}^{\beta-\mathrm{TRCP}} \mathrm{E} 3$ ubiquitin ligase, leading to YAP ubiquitination and degradation (Zhao et al. 2010b). Drosophila Yki is also inhibited by phosphorylation-induced translocation (Dong et al. 2007). Furthermore, Yki WW domains could interact with the PPXY motifs in Ex, Wts, and Hpo. Such interactions may inhibit Yki by phosphorylation-independent cytoplasmic sequestration, adding another layer of complexity to Yki regulation (Badouel et al. 2009; Oh et al. 2009).
Here we report the identification of angiomotin (AMOT) family proteins as strong interacting partners of YAP and TAZ (transcriptional coactivator with PDZ-binding motif) by tandem affinity purification (TAP) and mass spectrometry. The interaction maps to the WW domains of YAP and TAZ and the PPXY motifs of AMOT. Binding to AMOT family proteins is crucial to the tight junction localization of YAP and TAZ, and is also important for their phosphorylation by the Hippo pathway. Consistently, knockdown of AMOTL2 in Madin-Darby canine kidney (MDCK) cells leads to loss of tight junction localization and nuclear accumulation of both YAP and TAZ, induction of YAP/ TAZ target gene expression, and, importantly, loss of cell contact inhibition. Furthermore, AMOTL2 knockdowninduced foci formation is blocked by simultaneous knockdown of YAP and TAZ. Our results demonstrate AMOT family proteins as new components of the Hippo pathway with tumor-suppressing potential, and indicate a new mode of YAP and TAZ regulation.

\section{Results}

\section{YAP strongly interacts with AMOT family proteins}

To further elucidate the regulation and function of YAP, we carried out TAP of Flag-Streptavidin-binding peptide (SBP)-tagged YAP ectopically expressed in MCF10A cells. As shown in Figure 1A, many proteins specifically interact with YAP. To identify these proteins, total eluates were subjected to mass spectrometry analysis that identified many known YAP-binding proteins, including Lats1, TEAD1, and TEAD3 (Fig. 1B). Among novel YAPinteracting proteins were AMOTL1 and AMOTL2, two AMOT family proteins. Notably, both of them are on the
A

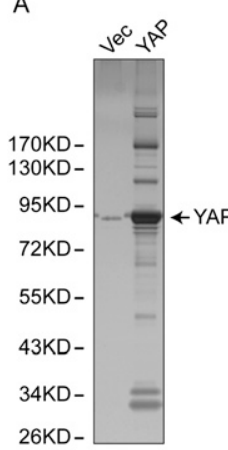

\begin{tabular}{|c|c|c|c|}
\hline Protein & Total peptides & $\begin{array}{c}\text { Unique } \\
\text { peptides }\end{array}$ & AA coverage \\
\hline YAP & 143 & 34 & $57.1 \%$ \\
\hline AMOTL2 & 44 & 38 & $55.1 \%$ \\
\hline AMOTL1 & 41 & 33 & $39.2 \%$ \\
\hline Lats1 & 9 & 9 & $12.5 \%$ \\
\hline TEAD1 & 3 & 3 & $11.0 \%$ \\
\hline TEAD3 & 2 & 1 & $4.7 \%$ \\
\hline
\end{tabular}

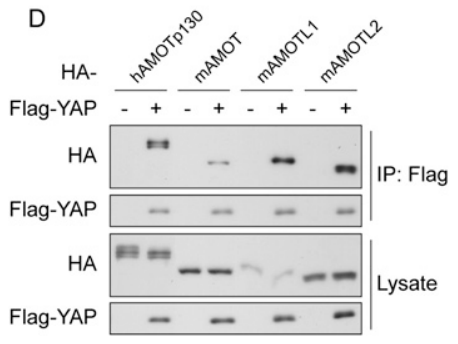

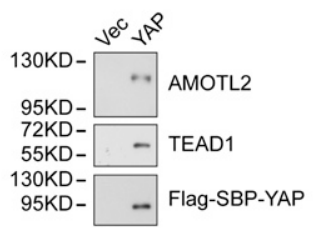

E

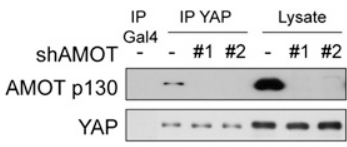

YAP

Figure 1. Identification of AMOT family proteins as YAP-binding partners. (A) Analysis of YAP TAP products. MCF10A cells stably expressing either the vector control or Flag-SBP-tagged YAP were established. Cells were grown to $70 \%$ confluence, and cell lysates were purified first with anti-Flag resin and then with streptavidin resin. The final eluates were resolved on SDS-PAGE gels and silver-stained. A weak band at the same position as YAP in the Vec lane is due to a leak from the YAP lane. $(B)$ Mass spectrometry analysis of YAP TAP products identified AMOT family members as strong interacting proteins. YAP TAP products were analyzed by MS-MS. The number of peptide hits for AMOTL1, AMOTL2, and several known YAP-interacting proteins are shown. (C) Confirmation of YAP-interacting proteins by Western blotting. Vec and YAP purification products were analyzed by Western blots using specific antibodies against AMOTL2, TEAD1, and Flag. $(D)$ All AMOT family proteins interact with YAP. YAP was cotransfected with human or mouse AMOT, AMOTL1, or AMOTL2 into HEK293 cells. YAP was immunoprecipitated with anti-Flag antibody, and coimmunoprecipitated AMOT family proteins were analyzed by anti-HA Western blot. (E) Endogenous YAP and AMOT interact in HEK293T cells. YAP was immunoprecipitated from cell lysates from normal HEK293T cells or two AMOT shRNA knockdown clones. AMOT p130 was detected by a specific antibody. Immunoprecipitation with anti-Gal4 antibody was used as a negative control. 
top of the peptide hit list-ahead of Lats1, TEAD1, and TEAD3-suggesting a strong interaction between AMOT family proteins and YAP. AMOT is known to associate with tight junction proteins, including Pals1 and PATJ (Wells et al. 2006). Interestingly, we also observed several tight junction proteins-including Pals1, PATJ, MPDZ, and Lin7C- that were copurified with YAP (data not shown), possibly mediated by AMOT family proteins. The presence of AMOTL2 and TEAD1 in YAP purification was confirmed by Western blots (Fig. 1C). We also performed TAP of TAZ from HEK293T cells and identified AMOT and AMOTL1 as top TAZ-associating proteins (Supplemental Fig. S1A).

The AMOT family has three paralogs in humans and mice: AMOT, AMOTL1, and AMOTL2. They share similar domain organization, including PPXY motifs, a coiled-coil domain, and a C-terminal PDZ-binding motif (Supplemental Fig. S1B). When cotransfected with YAP, the human AMOT p130 isoform (the longer one among two AMOT isoforms), and mouse AMOT, AMOTL1, and AMOTL2 all efficiently coimmunoprecipitated with YAP (Fig. 1D). To facilitate further study of endogenous proteins, we examined the expression of AMOT family members in several cell lines. Quantitative RT-PCR showed different expression patterns of the three AMOT genes, with at least one of them expressed in each cell line (Supplemental Fig. S1C). We also evaluated commercial antibodies against AMOT family proteins. The anti-AMOT antibody recognized only the human AMOT p130 isoform, such as in HEK293T cells, but detected no signal in NIH-3T3 mouse fibroblast cells, which also express high levels of AMOT mRNA (Supplemental Fig. S1C,D). The anti-AMOTL1 antibody detected only the protein from the mouse origin (Supplemental Fig. S1D). The anti-AMOTL2 antibody detected high background, which made it difficult to distinguish a specific AMOTL2 band (data not shown), although it detected highly concentrated AMOTL2 in TAP of YAP. Due to these restraints, we examined endogenous YAP and AMOT interaction in HEK293T cells. Quantification of Western blots indicated that immunoprecipitation with anti-YAP, but not an unrelated antibody, recovered $22 \%$ of YAP as well as $9 \%$ of endogenous AMOT. This converts to $\sim 40 \%$ of endogenous AMOT in association with YAP (Fig. 1E). shRNA knockdown of AMOT eliminates it from YAP immunoprecipitation. The above results have established AMOT family proteins as strong interacting partners of YAP under physiological conditions.

\section{The YAP WW domains and AMOT PPXY motifs mediate their interaction}

We next mapped the region responsible for YAP and AMOT interaction. AMOT has two isoforms generated by alternative splicing (Ernkvist et al. 2006). The p80 isoform lacks the $\mathrm{N}$-terminal 400 amino acids present in the p130 isoform (Fig. 2A). In a reciprocal coimmunoprecipitation experiment, AMOT p130, but not AMOT p80, showed clear interaction with YAP (Fig. 2B). Furthermore, when GST-YAP purified from Escherichia coli was in- cubated with HA-AMOT p130 or p80 immunoprecipitated from HEK293 cells, it interacted only with AMOT p130, but not the p80 (Supplemental Fig. S2). The p130 unique $\mathrm{N}$-terminal region contains two PPXY motifs (Fig. 2A), which could serve as binding partners for the YAP WW domains. Interestingly, mutation of the first PPXY motif, which is conserved in all three AMOT family members, significantly decreased interaction with YAP. Mutation of the second PPXY motif, which is not conserved in AMOTL2, had little effect (Fig. 2C). Combinatory mutation of both PPXY motifs showed an effect similar to mutation of the first PPXY motif (Fig. 2C). We further tested deletion of several other regions on AMOT p130 for the effect on YAP interaction. Deletion of the PDZ-binding motif, which is known to interact with PATJ (Wells et al. 2006), or the linker region, which is responsible for angiostatin binding in the p80 isoform (Troyanovsky et al. 2001), did not affect AMOT interaction with YAP. However, deletion of the coiled-coil domain also significantly attenuated interaction with YAP (Fig. 2D). The coiled-coil domain of AMOT interacts with Rich1 and is possibly involved in AMOT dimerization and localization (Wells et al. 2006; Ernkvist et al. 2008). Therefore, the coiled-coil domain may affect AMOT-YAP interaction indirectly. Taken together, both the PPXY motif and the coiled-coil domain of AMOT contribute to interaction with YAP.

WW domains are known to interact with PPXY motifs. Mutation of the first WW domain of YAP largely abolished YAP-AMOT interaction, while mutation of the second WW domain also decreased the interaction, albeit less potently (Fig. 2E). Mutation of both WW domains abolished the interaction similarly to mutation of the first WW domain (Fig. 2E). Noteworthy, mutation of YAP WW domains has a more profound effect on diminishing YAPAMOT interaction than mutation of AMOT PPXY motifs. One possibility is that endogenous AMOT may mediate YAP-AMOT-Y1Y2 interaction by dimerization with AMOT-Y1Y2. Alternatively, mutation of the tyrosine in PPXY motifs may not completely disrupt interaction with WW domains. Similar to YAP, TAZ also interacted with AMOT p130, but not p80, in a WW domain-dependent manner (Fig. 2F).

The YAP-AMOT interaction was further confirmed in live cells by fluorescence resonance energy transfer (FRET) experiments. In cells expressing YFP-AMOT p130 and CFP-YAP, bleaching of the FRET acceptor YFP caused a decrease of FRET, as visualized by a decreasing YFP emission with a concomitant rising of fluorescence emission from the FRET donor CFP (Fig. 2G). This supports a specific interaction between CFP-YAP and YFP-AMOT in live cells. However, if a CFP-YAP-W1W2 mutant was used, no significant increase of CFP signal was seen when YFP was bleached (Fig. 2G), indicating lack of FRET, hence no interaction between the two proteins. Similar results were observed when we switched the YFP and CFP tags on YAP and AMOT (data not shown). The above results showed that the WW domains of YAP and the first PPXY motif of AMOT play major roles in YAP-AMOT interaction. 


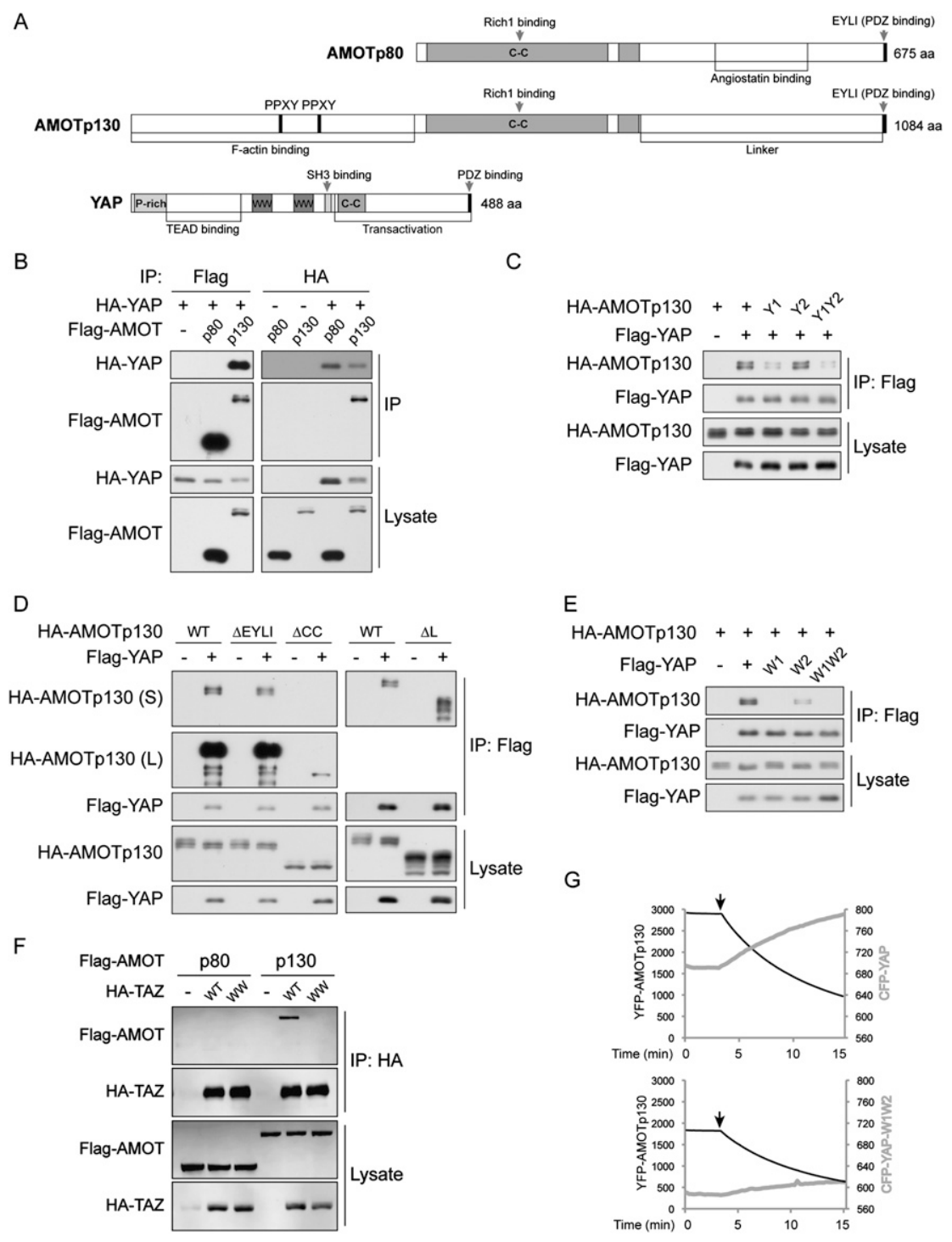

Figure 2. YAP WW domains interact with the AMOT PPXY motif. (A) Domain organization of AMOT isoforms and YAP. Human AMOT p80 and p130 splicing variants and YAP are drawn in scale. (C-C) Coiledcoil domain; (P-rich) proline-rich domain; (WW) WW domain. (B) AMOT p130 but not the $\mathrm{p} 80$ isoform interacts with YAP. YAP and AMOT p130 or p80 isoforms were cotransfected into HEK293 cells. AMOT or YAP was immunoprecipitated with antiFlag or anti-HA antibodies. Coimmunoprecipitation of the other protein was detected by respective Western blots as indicated. $(C)$ The first PPXY motif in AMOT is important for YAP interaction. AMOT p130 wild type or PPXY motif mutants (mutate to PPXA) were cotransfected with YAP into HEK293 cells. YAP was immunoprecipitated, and the coimmunoprecipitated AMOT p130 was examined by anti-HA Western blot. Y1, Y2, and Y1Y2 denote AMOT p130 with mutation of the first, second, or both PPXY motifs. (D) The AMOT coiled-coil domain contributes to the interaction with YAP. Experiments were similar to that in $C$ except that deletion mutants of AMOT p130 PDZ-binding motif ( $\Delta$ EYLI), coiled-coil domain $(\Delta \mathrm{CC})$, or linker region $(\Delta \mathrm{L})$ were used. $(E) \mathrm{WW}$ domains of YAP interact with AMOT. Experiments were similar to that in $C$ except that YAP first WW domain mutant (W1), second WW domain mutant (W2), or both WW domains mutant (W1W2) were used. $(F)$ The WW domain of TAZ interacts with AMOT p130. TAZ and AMOT p130 or p80 isoforms were cotransfected into HEK293 cells. TAZ was immunoprecipitated with anti-HA antibody. Coimmunoprecipitation of AMOT was detected by anti-Flag Western blot. $(G)$ YAP but not the W1W2 mutant interacts with AMOT p130 in live cells. YFP-AMOT p130 was cotransfected with CFP-YAP wild type or W1W2 mutant into Cos-7 cells. The YFP and CFP signal from a single cell is recorded every 15 sec. After $3 \mathrm{~min}$ (indicated by arrows), YFP was bleached before every recording.

\section{AMOT regulates YAP subcellular localization}

The Hippo pathway inhibits YAP at least in part by affecting YAP subcellular localization (Zhao et al. 2007). We examined whether YAP localization is also regulated by AMOT. In low-density HeLa cell culture, Flag-YAP was localized mainly in the cell nucleus (Fig. 3A). Coexpression of Mst2 and Lats2 induced translocation of YAP to cytoplasm. Interestingly, when AMOT p130 was coexpressed, Flag-YAP was also localized predominantly in the cytoplasm (Fig. 3A), indicating a role of AMOT in regulating YAP subcellular localization. However, localization of the YAP-W1W2 mutant was not affected by AMOT (Fig. 3A), supporting the importance of the interaction with AMOT in modulating YAP localization. The Hippo pathway affects YAP localization via phosphorylation-dependent 14-3-3 binding (Zhao et al. 2007). Mutation of the Hippo pathway phosphorylation sites makes YAP constitutively active, as shown in Figure 3A. The YAP-5SA mutant showed a more discrete nuclear localization than the wild-type protein and was no longer regulated by Lats (data not shown). However, ectopic expression of AMOT induced a significant cytoplasmic localization of YAP-5SA (Fig. 3A). This is consistent with the observation that YAP5SA retains interaction with AMOT (Supplemental Fig. S3A). The above data indicate a role of AMOT in stimulating YAP cytoplasmic localization. Under condition of AMOT p130 ectopic expression, the cytoplasmic translocation of YAP does not require phosphorylation by the Hippo pathway, suggesting the involvement of physical binding in this process.

AMOT p130 was reported to colocalize with F-actin and promote the formation of actin fibers in endothelial cells (Ernkvist et al. 2006). Consistently, in HeLa cells, AMOT 
A

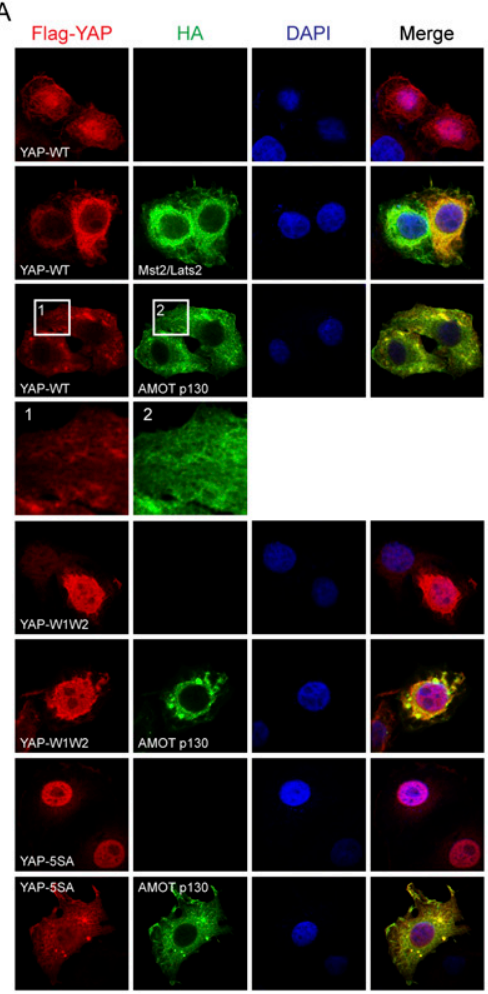

B
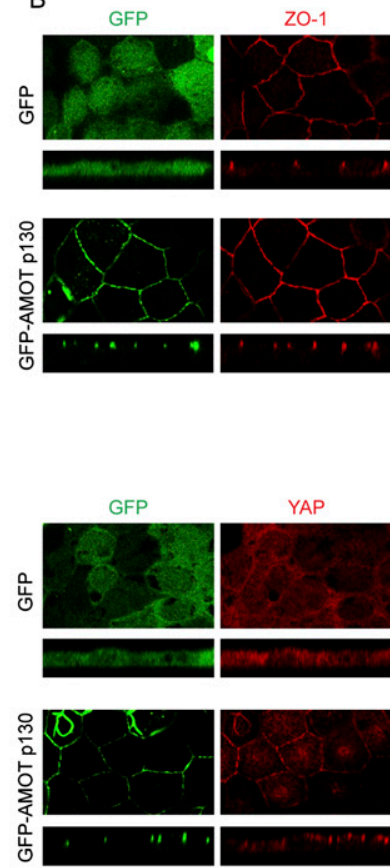
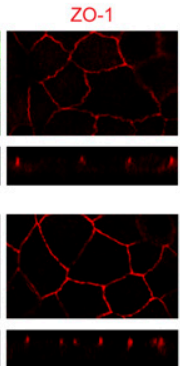

Merge
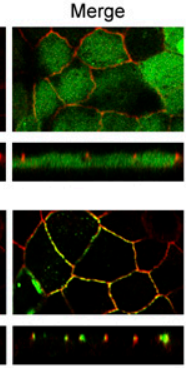

Merge
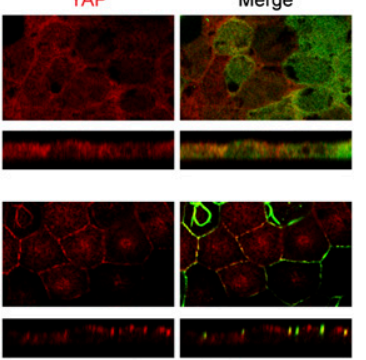

Figure 3. AMOT binding affects YAP subcellular localization. (A) AMOT alters YAP localization in HeLa cells. Flag-YAP wild type or mutants were transfected into HeLa cells with or without cotransfection of HAAMOT p130, or a combination of HA-Mst2/ HA-Lats2. Immunofluorescent stainings were done with anti-Flag and anti-HA antibodies. DAPI stains cell nuclei. An area from Flag-YAP-WT and HA-AMOT p130 expressed cells was enlarged to show the cytoskeleton-like localization of both proteins. (B) AMOT p130 promotes YAP localization to tight junctions in MDCK cells. MDCK cells infected with control GFP or GFPAMOT p130 were cultured onto transwell membranes. Polarized cells were fixed and stained with anti-ZO-1 tight junction marker or anti-YAP (from Dr. Marius Sudol's laboratory) for endogenous YAP localization. Both the top view and $Z$-stack view images are shown.

expression induced a meshwork-like localization of YAP similar to actin cytoskeleton (Fig. 3A). Such a change of YAP localization was more obvious in GFP-AMOT p130expressing MCF10A cells, in which GFP-AMOT p130 colocalized with bundled F-actin visualized by phalloidin staining (Supplemental Fig. S3B). Interestingly, in similar structures, we also observed colocalization of GFP-AMOT p130 with enriched endogenous YAP (Supplemental Fig. S3B). These observations indicate the ability of AMOT p130 to direct YAP localization to specific cell compartments.

In tight junction-forming epithelial cells such as MDCK cells, AMOT has been reported to localize to tight junctions (Wells et al. 2006). Indeed, we observed nice colocalization of GFP-AMOT p130, but not the GFP control, with tight junction protein ZO-1 in polarized MDCK cells (Fig. 3B). We next examined YAP localization in these cells. Using a previously reported anti-YAP antibody (Oka et al. 2008), we observed that, in well-polarized GFP-expressing MDCK cells, YAP localized mainly to the cytoplasm. However, expression of GFP-AMOT p130 brought a fraction of endogenous YAP to discrete tight junction localization (Fig. 3B). Specificity of the antibody was confirmed by loss of immunofluorescence staining in YAP knockdown cells (Supplemental Fig. S3C). The translocation of YAP was further confirmed by biochemical fractionation. Ectopically expressed GFP-YAP was recovered mainly in the cytoplasmic fraction from confluent MDCK cells, while GFP-AMOT p130 was mainly in the membrane fraction (Supplemental Fig. S3D), consistent with its tight junction localization. Interestingly, coexpression of AMOT p130 relocalized a significant fraction of GFP-YAP but not the YAP-W1W2 mutant to the cell membrane (Supplemental Fig. S3D). These data indicate that a direct interaction with AMOT contributes to the tight junction and cell membrane localization of YAP.

We then examined whether endogenous YAP localizes to tight junctions under physiological conditions. However, by staining MDCK cells with the antibody used above, we could not observe specific localization of YAP to tight junctions in untransfected MDCK cells, which could be due to the low level of tight junction-associated YAP or the lack of binding of the antibody to the tight junctionassociated YAP. Therefore, we tested a new antibody that was raised against the YAP C-terminal region, which is not involved in AMOT binding. Furthermore, we also modified the immunofluorescence staining protocol by pretreating cells with detergent before fixation to remove freely diffusible YAP. With these improvements, we indeed observed that a fraction of endogenous YAP colocalized with the tight junction marker ZO-1 in normal MDCK cells, although the signal was weak (Fig. 4A). The specificity of this anti-YAP antibody was confirmed by the loss of Western blot signals and tight junction immunofluorescence staining in YAP knockdown MDCK cells (Supplemental Fig. S4A).

To determine the function of endogenous AMOT family proteins in controlling YAP tight junction localization, we examined AMOTL2 knockdown in MDCK cells (Fig. 5D), which express a high level of AMOTL2 but a low level of AMOT (Supplemental Fig. S1C). Notably, in AMOTL2 knockdown cells, a clear loss of tight junction-localized YAP was observed, and an accompanied 


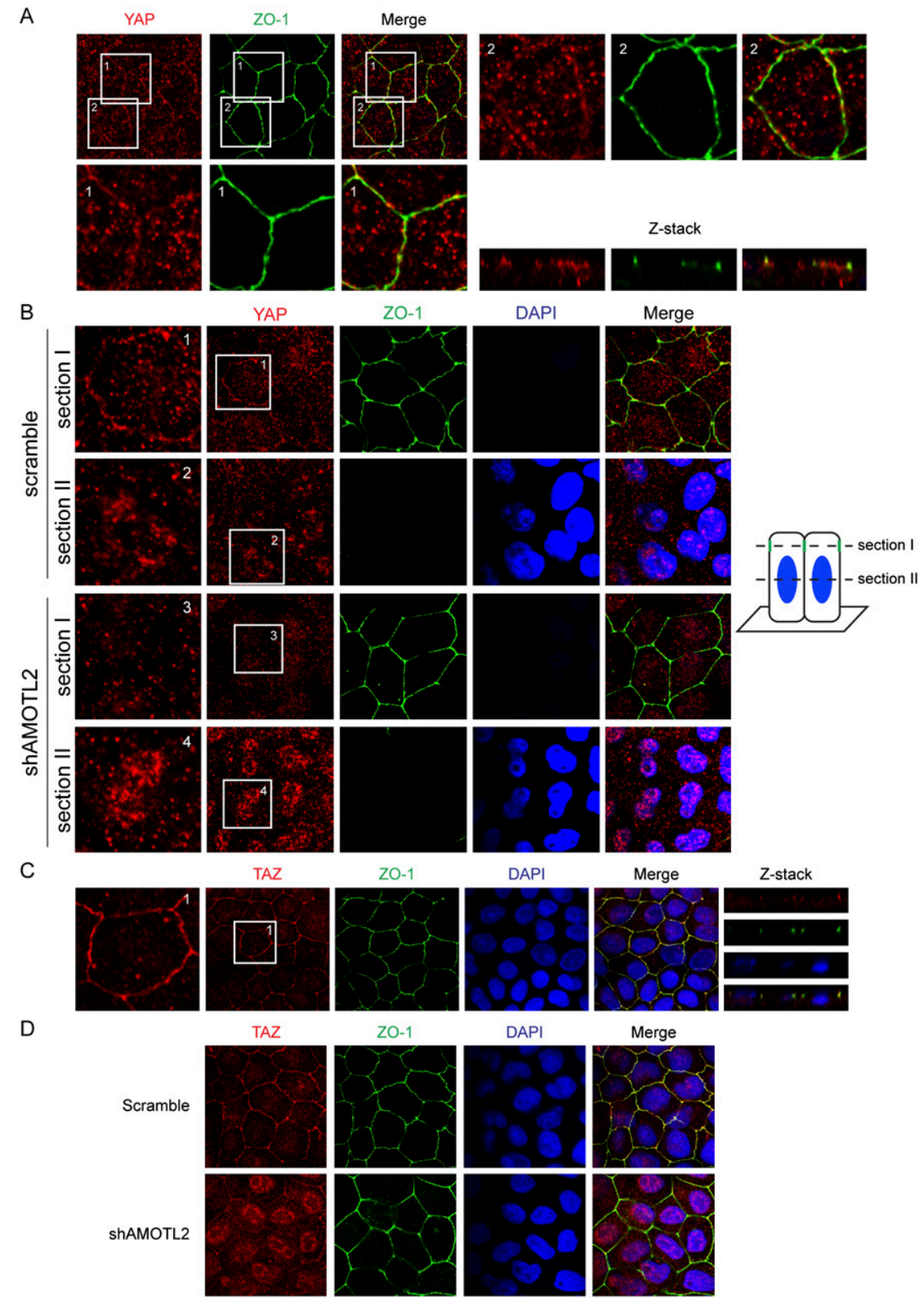

Figure 4. AMOTL2 is required for YAP and TAZ tight junction localization in MDCK cells. (A) A fraction of endogenous YAP localizes to tight junctions in MDCK cells. Confluent MDCK cells were treated with $1 \%$ Triton for $5 \mathrm{~min}$ and then fixed and stained with anti-ZO-1 or anti-YAP (from Bethyl Laboratories). Enlarged views and a $Z$-stack view are shown. YAP and ZO-1 were stained as red and green, respectively. (B) AMOTL2 knockdown leads to loss of tight junction-localized YAP and accumulation of nuclear YAP. Scramble and AMOTL2 knockdown MDCK cells were established by lentiviral infection of MDCK cells with shRNA constructs. Staining is similar to that in $A$, and for each field images were acquired on two optical sections as indicated. Section I was set at the position of the tight junction, whereas section II was set at the position of the nucleus. $(C)$ Tight junction localization of TAZ in MDCK cells. MDCK cells were stained using anti-TAZ antibody in a procedure similar to that in $A$. (D) AMOTL2 knockdown leads to loss of tight junction-localized TAZ and accumulation of nuclear TAZ. The same cells as that in $B$ were used for TAZ staining, and the thickness of the optical slice covers both the cell nuclear and apical regions. increase of nuclear-localized YAP was also observed (Fig. 4B). Furthermore, when a similar staining procedure was used to stain TAZ in MDCK cells using a validated TAZ antibody (Supplemental Fig. S4B), we observed a distinct tight junction localization of endogenous TAZ (Fig. 4C). Similar to what was observed for YAP, AMOTL2 knockdown in MDCK cells also led to a loss of tight junction-localized TAZ and an accumulation of nuclear TAZ (Fig. 4D). The above results indicate that, in MDCK cells, a fraction of endogenous YAP/TAZ localizes to tight junctions in an AMOT family proteindependent manner. Loss of YAP/TAZ tight junction localization caused by AMOTL2 knockdown leads to their nuclear accumulation and possibly activation, supporting a physiological role of AMOTL2 in regulation of YAP/TAZ.
AMOT p130 promotes YAP phosphorylation and inhibits YAP transcriptional activity

Since AMOTL2 knockdown results in YAP/TAZ nuclear accumulation, which is known to be regulated also by phosphorylation, we examined whether AMOTL2 knockdown altered YAP/TAZ phosphorylation. Indeed, we observed a drop of YAP and TAZ phosphorylation as determined by phosphorylation-specific antibodies and differential migration on Phos-tag gel, which specifically retards phosphorylated proteins (Fig. 5A). This result suggests that decreased YAP/TAZ phosphorylation might contribute to the YAP/TAZ nuclear accumulation observed in AMOTL2 knockdown cells.

We further examined the physiological role of AMOT family proteins in YAP phosphorylation in additional cell 
A
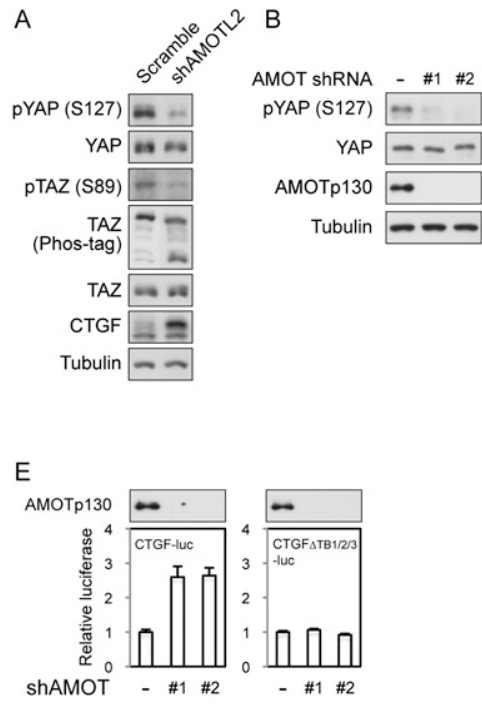

C

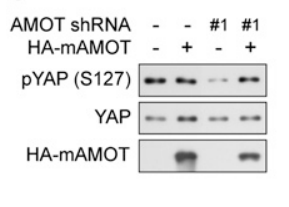

D

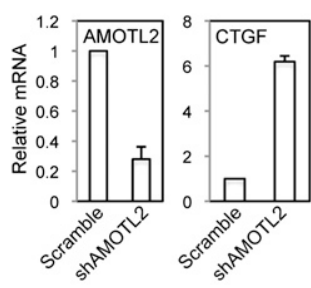

F

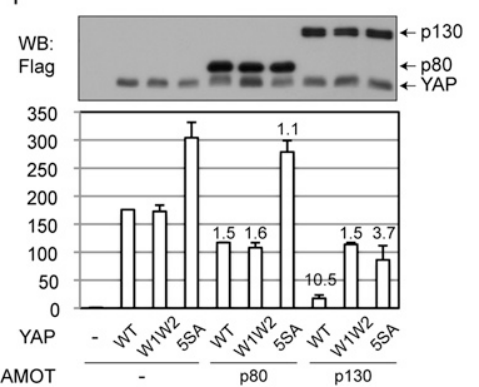

Figure 5. AMOT family proteins are important for YAP/TAZ phosphorylation and inhibit YAP/TAZ transcription coactivator activity. (A) AMOTL2 promotes YAP and TAZ phosphorylation in MDCK cells. Scramble and AMOTL2 knockdown MDCK cells were established by lentiviral infection of MDCK cells with shRNA constructs. Cell lysates were examined by Western blots using indicated antibodies. The antitubulin Western blot was used as a loading control. $(B)$ Knockdown of AMOT in HEK293T cells attenuated YAP phosphorylation. YAP phosphorylation levels were examined in two clones of AMOT knockdown HEK293T cells by Western blots. $(C)$ Re-expression of AMOT in AMOT knockdown cells rescues YAP phosphorylation. Mouse AMOT was transfected into control or AMOT knockdown HEK293T cells, and YAP phosphorylation levels were shown by Western blots. (D) AMOTL2 knockdown in MDCK cells induces CTGF expression.

RNA was extracted from cells the same as those in $A$, and mRNA levels of AMOTL2 and CTGF were examined by quantitative RT-PCR. $(E)$ Endogenous AMOT inhibits the activity of a YAP reporter. Control or AMOT knockdown HEK293T cells were transfected with CTGF-luciferase reporter and the CMV- $\beta$-gal control. Luciferase activity was measured and normalized to $\beta$-galactosidase activity. $\mathrm{CTGF}_{\Delta \mathrm{TB} 1 / 2 / 3}$-luc indicates the CTGF-luciferase reporter with mutation of all three TEAD-binding sites. AMOT p130 expression levels were determined by Western blots. (F) AMOT p130 inhibits YAP in luciferase assay. Indicated plasmids were cotransfected with a $5 \times$ UAS-luciferase reporter, Gal4-TEAD4, and CMV- $\beta$-gal constructs into HEK293T cells. Luciferase activity was measured and normalized to $\beta$-galactosidase activity. Folds of YAP inhibition by AMOT were labeled on top of columns. YAP and AMOT expression levels were determined by anti-Flag Western blot.

types. In HEK293T cells, AMOT expression is high and AMOTL2 expression is low (Supplemental Fig. S1C). AMOT knockdown in this cell line also led to a loss of membrane-localized YAP (Supplemental Fig. S5A) and attenuated YAP phosphorylation on S127, a Hippo pathway target site (Fig. 5B). The effect of AMOT knockdown on YAP phosphorylation was rescued by re-expression of a shRNA-resistant mouse AMOT (Fig. 5C). AMOT overexpression did not increase YAP phosphorylation in control HEK293 cells (Fig. 5C). This could be due to the high level of endogenous AMOT in this cell line or the limited amount of another factor required for AMOT to stimulate YAP phosphorylation in HEK293 cells. These results indicate that endogenous AMOT family proteins are important for YAP phosphorylation by the Hippo pathway.

Consistently, we also observed activation of YAP and TAZ caused by knockdown of AMOT family proteins. In AMOTL2 knockdown MDCK cells, expression of CTGF, a direct YAP and TAZ target gene, was increased at both protein and mRNA levels (Fig. 5A,D). In HEK293T cells, AMOT knockdown consistently activated a CTGF promoter reporter (Fig. 5E). As a negative control, a mutant CTGF reporter with all three TEAD-binding sites disrupted was insensitive to AMOT knockdown, indicating that the increased CTGF promoter activity in AMOT-deficient cells was due specifically to increased YAP-TEAD function. Moreover, expression of AMOT p130 strongly inhibited (a 10.5-fold reduction) YAP activation of Gal4-TEAD4 on the $5 \times$ UAS-luciferase reporter (Fig. 5 F). In contrast, the YAP-W1W2 mutant was not efficiently inhibited by
AMOT p130 (Fig. 5F). AMOT p80 also had little effect on either YAP wild type or the W1W2 mutant. As we reported previously, the YAP-5SA mutant, which could not be phosphorylated by the Hippo pathway, has elevated activity. This mutant was partially inhibited (3.7-fold) by coexpression of AMOT p130 (Fig. 5F), supporting that both Hippo pathway phosphorylation-dependent and -independent mechanisms are involved in YAP inhibition by AMOT p130. In addition, coexpressed AMOT p130 and Hippo pathway proteins cooperated to produce a greater degree of YAP inhibition (Supplemental Fig. S5B). This inhibition likely did not involve a direct competition of AMOT p130 with TEAD because coimmunoprecipitation of YAP and TEAD1 was not affected by the presence of ectopically expressed AMOT p130 (Supplemental Fig. S5C). The above data support AMOT as a physiological inhibitor of YAP transcription coactivator activity.

\section{AMOT inhibits YAP-induced transformation and loss of cell contact inhibition in the epithelial sheet}

We then set out to determine the role of AMOT family proteins in regulating YAP cellular function by first establishing a functional assay for YAP in MDCK cells. We observed that cells infected with GFP-YAP formed foci (Fig. 6A). These foci were further analyzed by confocal microscopy after rhodamine-phalloidin staining for actin cytoskeleton to mark cell boundaries. While control GFP cells integrated seamlessly in the monolayer epithelial sheet, GFP-YAP cells were extruded from the GFP-negative 


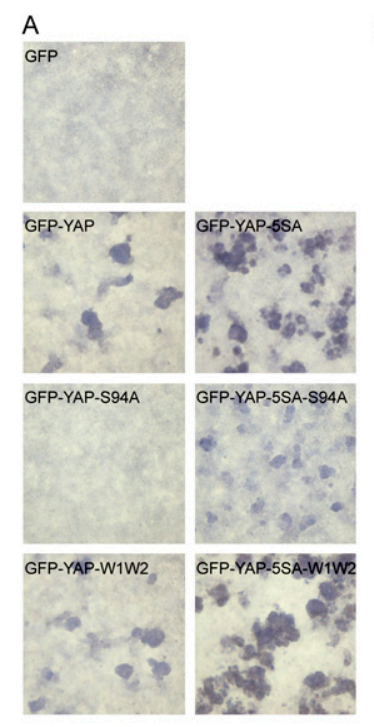

C

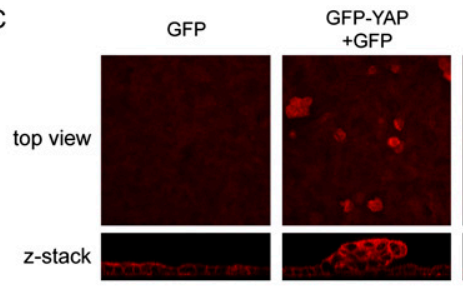

D

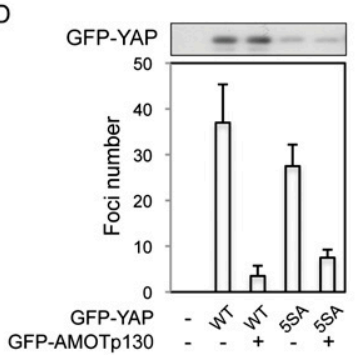

B

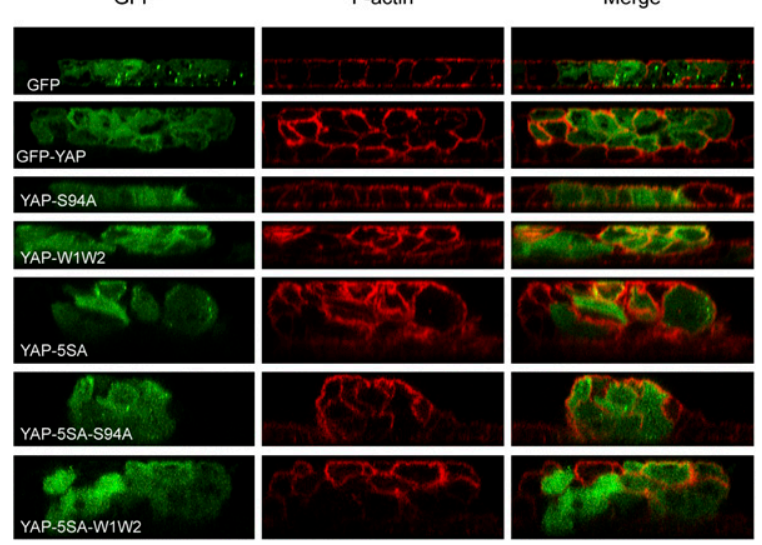

Figure 6. AMOT inhibits the activity of YAP in inducing transformation and loss of cell contact inhibition in the epithelial sheet. (A) YAP induces MDCK cell foci formation. MDCK cells infected with GFP control or GFP-YAP wild type or mutants were cultured onto transwell membranes at a density of 1 million cells per 24-mm insert. Cells were further cultured for $5 \mathrm{~d}$, and cell foci were visualized by Giemsa stain. (B) MDCK cell foci induced by YAP are multilayer cells showing loss of contact inhibition. The same cells as in $A$ were fixed and stained with rhodamine-phalloidin to visualize F-actin. $Z$-stack views of confocal microscopy images were presented. $(C)$ AMOT inhibits YAP-induced MDCK cell foci formation. MDCK cells infected with GFP-YAP or GFP-YAP-5SA were reinfected with control GFP or GFP-AMOT p130. Cells were then cultured onto transwell membranes for foci formation assay. Cells were fixed and stained with rhodaminephalloidin. Both lower-magnification top views and higher-magnification $Z$-stack images were shown. (D) Quantification of AMOT effect on YAP-induced MDCK foci formation. Cell foci in five random views were counted. GFP-YAP expression levels were shown by anti-YAP Western blot. $(E)$ AMOT inhibits YAP-induced CTGF expression in MDCK cells. MDCK cells infected as indicated were lysed, and cell lysates were analyzed for CTGF expression by Western blots.

monolayer and grew on top of each other to form multilayer foci (Fig. 6B). Remarkably, most GFP-YAP cells were excluded from the basal layer of cells that attached to the supporting membrane (Fig. 6B). A previous study showed that interaction with surrounding normal cells excludes transformed cells from the normal epithelial sheet, suggesting that the GFP-YAP cells are transformed (Hogan et al. 2009). These observations provide a convenient and robust functional assay for YAP, and demonstrate that high YAP activity overrides contact inhibition in epithelial cells. Furthermore, we observed that, when expressed at similar levels (Supplemental Fig. S6), YAP-5SA displayed higher activity than wild-type YAP in inducing foci formation (Fig. $6 \mathrm{~A}, \mathrm{~B})$, and that mutation of S94, which eliminates TEAD binding, in either the wild-type or 5SA background largely inhibited foci formation. These findings are consistent with previous reports that YAP function largely relies on TEAD binding and is inhibited by Hippo pathway-mediated phosphorylation (Zhao et al. 2007, 2008). The effect of WW domain mutation could be complicated because it has been implicated to play a positive role in YAP function by interacting with other transcription factors (X Zhang et al. 2009; Zhao et al. 2009), and the same domain also plays a role in YAP inhibition by binding Lats1/2 (Oka et al. 2008) and AMOT, as shown in this study.

After establishing the oncogenic activity of YAP in MDCK cells, we examined whether this activity is regulated by AMOT. Interestingly, expression of AMOT p130 largely decreased both cell focus number and layers of cells in each focus of GFP-YAP-expressing MDCK cells (Fig. 6C,D). YAP-5SA drove formation of larger foci, although when infection efficiency was controlled at a low level, a similar number of foci was formed. Consistent with the ability of AMOT to affect YAP-5SA localization and inhibit its activity, AMOT p130 also reduced YAP-5SA-induced foci formation (Fig. 6C,D), although the magnitude was less compared with the inhibition of wild-type YAP. Consistently, YAP-induced CTGF expression was also blocked by AMOT p130 coexpression (Fig. 6E). These observations have revealed the ability of AMOT to inhibit the oncogenic activity of YAP in MDCK cells, thus indicating a potential tumor suppressor function of AMOT. 
AMOTL2 knockdown induces MDCK cell transformation in a YAP-and TAZ-dependent manner

To test whether endogenous AMOT family proteins play a role in repressing the oncogenic activity of YAP, we examined AMOTL2 knockdown in MDCK cells in foci formation assays. Remarkably, AMOTL2 knockdown alone in MDCK cells was sufficient to induce foci formation (Fig. 7A; Supplemental Fig. S7A). Many cells in the foci displayed elevated nuclear YAP (Fig. 7B). Because YAP was known to induce enlarged acini of MCF10A cells in threedimensional culture, we also tested whether AMOTL2 knockdown in MDCK cells could induce a similar phenotype. Indeed, AMOTL2 knockdown MDCK cells formed larger acini with filled lumen, whereas control acini were smaller with cleared lumen (Supplemental Fig. S7B). These results complement the data obtained with AMOT over- expression experiments and provide the first evidence for a tumor suppressor function of AMOT family proteins.

To determine whether YAP and TAZ play a role in AMOTL2 knockdown-induced transformation, we examined the effect of knockdown of YAP and TAZ in AMOTL2deficient cells. Knockdown of YAP or TAZ individually in AMOTL2 knockdown MDCK cells significantly reduced the expression of CTGF (Fig. 7C). Simultaneous knockdown of YAP and TAZ completely blocked the induction of CTGF caused by AMOTL2 knockdown. Consistently, individual knockdown of YAP or TAZ partially abolished AMOTL2 knockdown-induced foci formation (Fig. 7D), whereas double knockdown of YAP and TAZ fully blocked foci formation in AMOTL2 knockdown MDCK cells (Fig. 7D). In this assay, cells with simultaneous knockdown of AMOTL2, YAP, and TAZ are viable (Supplemental Fig. S7C), indicating that the elimination of foci was not caused by massive

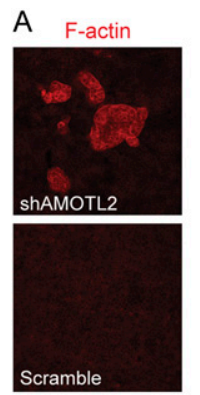

B

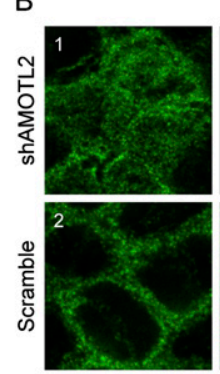

C

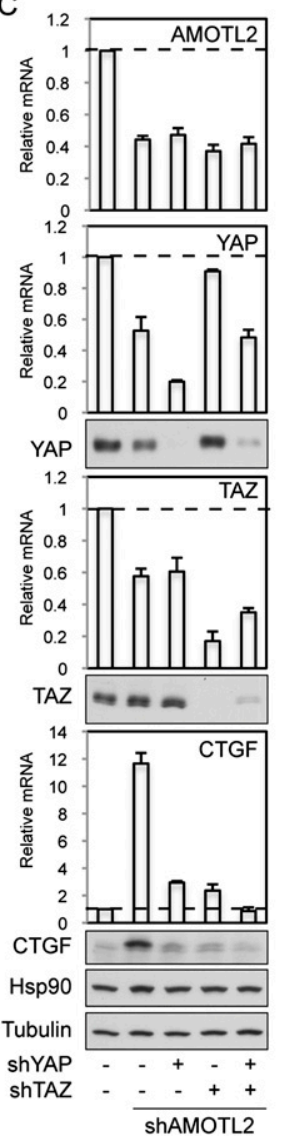

YAP

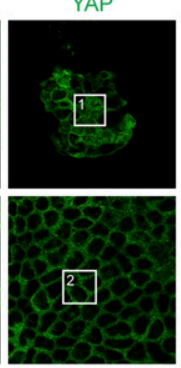

D

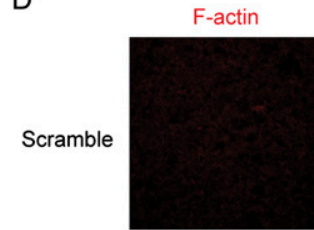

ShAMOTL2
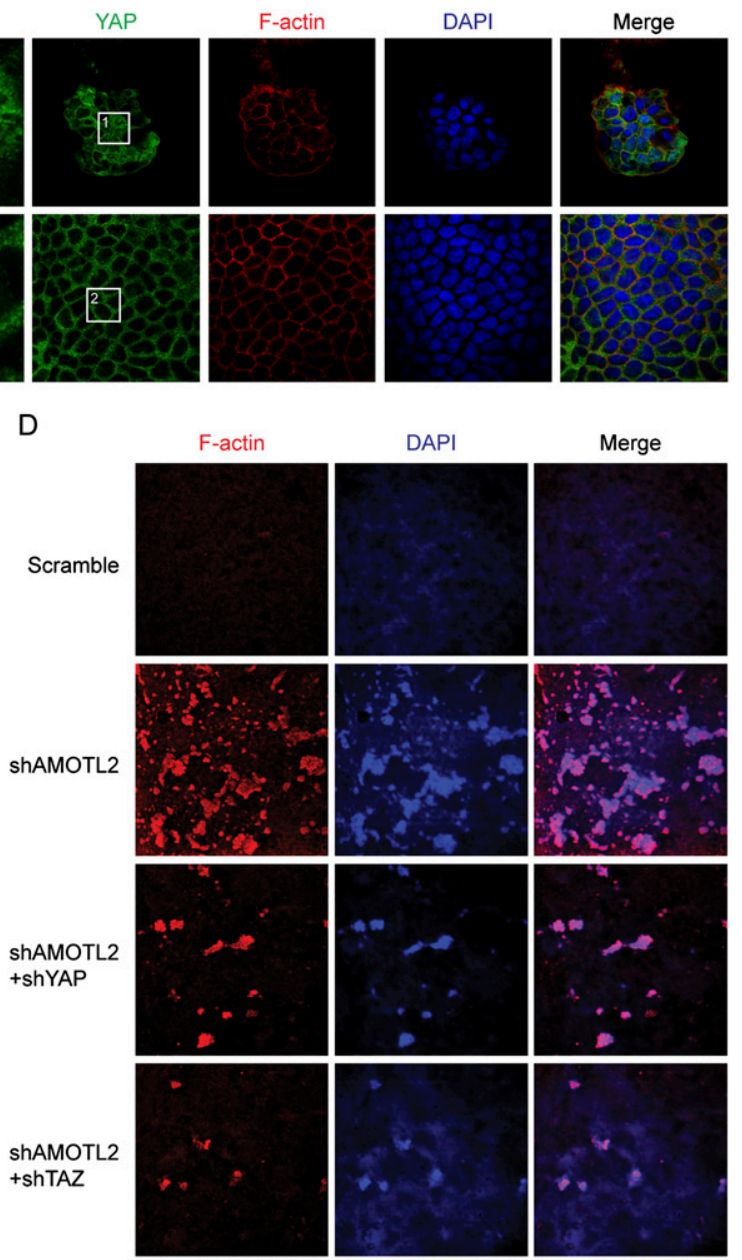

ShAMOTL2

+ shYAP

$+\operatorname{shTAZ}$
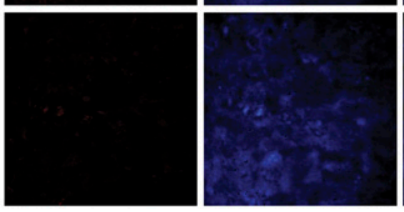

Figure 7. AMOTL2 knockdown induces cell transformation in MDCK cells in a YAP- and TAZ-dependent manner. (A) AMOTL2 knockdown in MDCK cells induces foci formation. Scramble and AMOTL2 knockdown MDCK cells were established by lentiviral infection of MDCK cells with shRNA constructs. Cells were then cultured onto transwell membranes at a density of 1 million cells per 24$\mathrm{mm}$ insert. After $5 \mathrm{~d}$, cell foci were visualized by rhodamine-phalloidin staining. $(B)$ AMOTL2 knockdown-induced cell foci have elevated nuclear YAP levels. Cells the same as those in $A$ were fixed and stained with anti-YAP (from Dr. Marius Sudol's laboratory) to visualize YAP localization, and with rhodamine-phalloidin to show cell boundaries. DAPI labels cell nuclei. Images of cells in shAMOTL2-induced foci above the monolayer (top panels) and of cells in the monolayer of control cells (bottom panels) are shown. Panels 1 and 2 represent insets from YAP staining as indicated. $(C)$ Knockdown of YAP and TAZ blocks AMOTL2 knockdowninduced CTGF expression. MDCK cells infected with indicated shRNA constructs were established. Levels of indicated mRNAs and proteins were analyzed by quantitative RTPCR and Western blots, respectively. Antitubulin and anti-Hsp90 Western blots were used as loading controls. (D) Knockdown of YAP and TAZ blocks AMOTL2 knockdowninduced foci formation. Cells the same as those in $C$ were tested in foci formation assays following the procedures in $A$. Cell foci were visualized by both rhodamine-phalloidin staining of F-actin and DAPI staining of cell nuclei. 
cell death. These data show that AMOTL2 knockdowninduced cell transformation depends on YAP and TAZ, and is consistent with a model in which AMOTL2 acts upstream and inhibits the function of YAP/TAZ.

\section{Discussion}

AMOT family proteins as new components of the Hippo pathway

YAP is a human oncogene and a potent promoter of organ size, which is negatively regulated by the Hippo pathway. Understanding the mechanisms of YAP regulation has significant implications in elucidating physiological organ size control and in therapeutics for human cancer. In this study, we identified AMOT family proteins as novel inhibitors of YAP. The AMOT family proteins strongly interact with YAP WW domains through their PPXY motifs and play an important role in YAP inhibition under physiological conditions. TAZ, the YAP paralog up-regulated in breast cancers (Chan et al. 2008), is regulated by AMOT family proteins in a similar fashion.

Our results indicate that AMOT can inhibit YAP/TAZ by two mechanisms (Fig. 8). Via physical interaction, AMOT recruits YAP/TAZ to various compartments such as tight junctions and actin cytoskeleton, depending on cellular context. This mechanism is independent of YAP/ TAZ phosphorylation by Lats1/2. In Drosophila, where an AMOT homolog is not present, Yki is inhibited partly through association with Expanded (Badouel et al. 2009; Oh et al. 2009). Therefore, an inhibition of YAP/TAZ/Yki by direct binding may be a common mechanism of regulation, although different genes are involved in Drosophila and mammalian cells. Endogenous AMOT family proteins also inhibit YAP/TAZ by promoting their inhibitory phosphorylation. AMOT family proteins could enhance YAP/TAZ phosphorylation. It is not yet clear whether AMOT enhances YAP phosphorylation indirectly by modulating YAP subcellular localization; for example, to the tight junction. Of note, Hippo pathway proteins Lats1, Mst1, and Drosophila Mats (Mob1 homolog) were reported to be activated

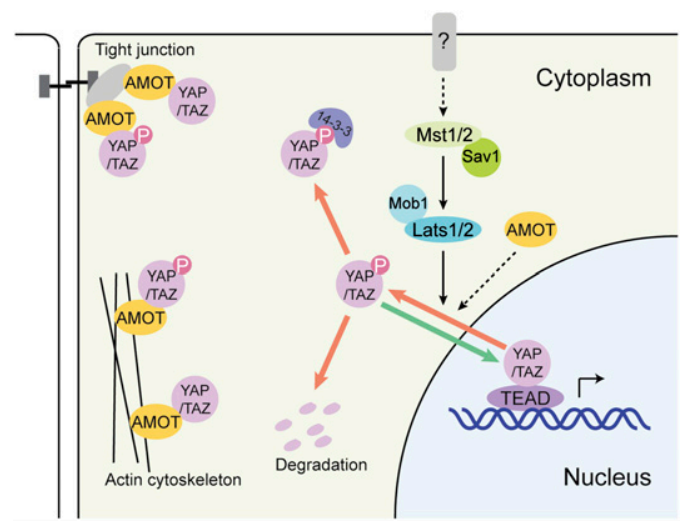

Figure 8. A model of AMOT in YAP/TAZ regulation. In this model, AMOT inhibits YAP/TAZ by regulating YAP/TAZ localization via physical interaction and promoting YAP/TAZ phosphorylation by the Hippo pathway. by membrane targeting (Avruch et al. 2006; Hergovich et al. 2006; Ho et al. 2009). Therefore, the tight junction might serve as a platform for Hippo pathway phosphorylation events to occur. Although not examined in this study, another possible mechanism of AMOT in inhibition of YAP biological functions is that AMOT binding to YAP WW domains directly competes with PPXY motif-containing transcription factors for YAP binding.

The Hippo pathway and AMOT may have overlapping and differential roles in YAP inhibition, dependent on cell context. AMOT family proteins are required for efficient YAP/TAZ inhibition by the Hippo pathway, at least in some cell contexts such as MDCK cells, in which knockdown of AMOTL2 leads to cell transformation and inhibition of YAP/TAZ phosphorylation despite the presence of Hippo pathway proteins. However, in some mouse tissues such as liver and spleen, none of the AMOT family proteins shows the significant expression determined by Northern blots in a previous study (Bratt et al. 2002). Therefore, it remains possible that, in some tissues, the Hippo pathway functions in an AMOT-independent manner. On the other hand, in certain organs such as lung, kidney, and intestines, knockout of the Hippo pathway kinases Mst1 and Mst2 does not have an obvious effect on organ size (Song et al. 2010). It is therefore possible that AMOT family proteins, through a physical binding mechanism, inhibit YAP/TAZ in these organs.

\section{AMOT family proteins as potential tumor suppressors}

AMOT was first identified as an angiostatin-binding protein promoting endothelial cell migration and angiogenesis (Troyanovsky et al. 2001). It has been implicated in tumor growth due to its angiogenic function. However, the angiostatin-responsive migration-promoting functions are observed only in the AMOT $\mathrm{p} 80$ splicing variant that does not bind YAP, but not in the YAP-binding p130 variant (Ernkvist et al. 2006). AMOT knockout mice display abnormal cell accumulation in extraembryonic tissue, attributed to defective cell migration (Shimono and Behringer 2003). Considering the differential functions of AMOT isoforms and possible functional redundancy of AMOT family members, the phenotypes observed might not accurately reflect the importance of AMOT in growth regulation. The identification of YAPs as AMOT p130binding proteins provides another link of AMOT to tumorigenesis. In MDCK epithelial cells, knockdown of AMOTL2 is sufficient to activate YAP/TAZ (partly by relieving inhibition by the Hippo pathway) and induce cell transformation. This is the first observation that AMOT family proteins could play a tumor suppressor role, at least in our cell culture models. A systematic analysis of possible changes in the AMOT family protein expression profile and mutation status will be needed to address the significance of AMOT family proteins in human cancer.

\section{AMOT as a possible connection between cell contact signals and YAP inhibition}

AMOT has been reported to play a role in tight junction integrity (Wells et al. 2006). The tight junction localization 
of AMOT involves the PDZ-binding motif, the coiled-coil domain, and also the $\mathrm{N}$-terminal region, which contains YAP/TAZ-binding sites. It was shown that the AMOT p130 N terminus itself could localize to tight junctions efficiently (Ernkvist et al. 2008). Therefore, it is possible that YAP and TAZ play a role in tight junction formation or maintenance by regulating AMOT.

YAP is regulated by cell density, and its inactivation plays a role in cell contact inhibition. However, the mechanism from cell contact sensing to YAP inhibition is elusive. Interestingly, several recent reports showed that two cell polarity protein complexes (including the apical tight junction proteins Crumbs [Crb] and aPKC, and the basolateral protein complex Scribble-Dlg-Lgl) modulate Hippo pathway activity in Drosophila (Grzeschik et al. 2010; Ling et al. 2010; Robinson et al. 2010). The function of these proteins in mammalian YAP regulation has not been demonstrated.

In this study, we show that fractions of endogenous YAP and TAZ adopt tight junction localization in polarized epithelial cells in an AMOT-dependent manner. We demonstrate that components of tight junction complexes inhibit YAP/TAZ. Our study suggests a more direct role of tight junction proteins in the regulation of YAP. For example, tight junction remodeling upon cell density change may affect AMOT localization and then regulate YAP localization and phosphorylation. In HEK293T cells, which lack functional tight junctions, we did not observe a differential coimmunoprecipitation of YAP and AMOT from cells cultured at different densities (Supplemental Fig. S8). Further testing of this possibility in other cell types is limited by the availability of proper anti-AMOT family protein antibodies. Further study is needed to demonstrate whether AMOT mediates the elusive contact signals, such as from tight junctions, to inhibit $\mathrm{YAP} / \mathrm{TAZ}$, and therefore to confer a contact inhibition in nontransformed cells.

\section{Materials and methods}

\section{Cell culture, transfection, and lentiviral infection}

HEK293 cells, HEK293T cells, HeLa cells, and MDCK cells were cultured in DMEM (Invitrogen) containing 10\% FBS (Invitrogen) and $50 \mu \mathrm{g} / \mathrm{mL}$ penicillin/streptomycin $(\mathrm{P} / \mathrm{S})$. Transfection with Lipofectamine (Invitrogen) was performed according to the manufacturer's instructions. For lentiviral infection, HEK293T cells were transfected with lentiviral constructs and packaging plasmids (psPAX2 and pMD2.G). Forty-eight hours after transfection, the lentiviral supernatant was supplemented with $5 \mu \mathrm{g} / \mathrm{mL}$ polybrene, filtered through a $0.45-\mu \mathrm{m}$ filter, and used to infect target cells.

\section{shRNA knockdown of AMOT and AMOTL2}

pSM2c-shRNAmir retroviral constructs targeting AMOT (clone ID: V2HS_76026) were purchased from Thermo Scientific Open Biosystems. The pLenti-lox-Amot-sh construct was generated by cloning annealed oligos 5 '-TGGTGGAATATCTCATCTAATTCA AGAGATTAGATGAGATATTCCACCTTTTTTC-3' and 5' '-TCG AGAAAAAAGGTGGAATATCTCATCTAATCTCTTGAATTA GATGAGATATTCCACCA-3' into the pLenti-lox-3.7 vector. Both constructs target human AMOT p80 and p130 isoforms.
HEK293T cells were infected with virus produced from both vectors. GFP-positive cells after infection were selected by flow cytometry, and two clones (\#1 and \#2) were used in this study.

To generate the dog AMOTL2 knockdown construct, the following oligonucleotides were cloned into pLKO.1 with the AgeI/ EcoRI sites: sense, CCGGGCGGGAGAAAGAGGAGCAAATCT CGAGATTTGCTCCTCTTTCTCCCGCTTTTTC; antisense, AA TTGAAAAAGCGGGAGAAAGAGGAGCAAATCTCGAGATT TGCTCCTCTTTCTCCCGC.

\section{Immunofluorescence staining}

MDCK cells were cultured on transwell supporting membranes, and other cells were cultured on coverslips to appropriate density. Cells were fixed with $4 \%$ paraformaldehyde for $15 \mathrm{~min}$ and then permeabilized with $0.1 \%$ Triton X-100. After blocking in 3\% BSA for $30 \mathrm{~min}$, slides were incubated with the first antibody diluted in $1 \%$ BSA for $1.5 \mathrm{~h}$. After washing with PBS, slides were incubated with Alexa Fluor 488- or 594-conjugated secondary antibodies (1:1000 dilution) for $1.5 \mathrm{~h}$. For staining of F-actin, cells were incubated with $6.6 \mathrm{nM}$ rhodamine-phalloidin for $1.5 \mathrm{~h}$ after blocking. The slides were then washed and mounted. For visualization of tight junction-localized YAP and TAZ, cells were first treated with detergent-containing buffer $(0.5 \%$ Triton X-100 in 68 mM PIPES, $25 \mathrm{mM}$ HEPES, $15 \mathrm{mM}$ EGTA, $3 \mathrm{mM} \mathrm{MgCl}_{2}, 10 \%$ dimethylsulfoxide at $\mathrm{pH}$ 6.8) for $5 \mathrm{~min}$, and then fixed and stained as described above.

\section{MDCK foci formation assay}

MDCK cells were infected with lentiviruses expressing GFP-YAP fusion proteins, or were further infected with GFP-AMOT constructs. Infected cells (1 million) were cultured onto 24-mm transwell inserts with $0.4-\mu \mathrm{m}$ pore size. Cells were further cultured for 3-6 d. Cell foci were visualized by staining with Giemsa stain or rhodamine-phalloidin.

\section{Acknowledgments}

We thank Drs. Chunling Yi and Joseph L. Kissil for AMOT knockdown constructs and AMOT knockdown HEK293T stable cells, Marius Sudol for an anti-YAP antibody, Xiao-Wei Chen for pHRCTS-CMV-WPRE-GFP vector, Philip Gafken at the Fred Hutchinson Cancer Center for assistance on LC-MS/MS analyses, Alyssa Wu for assistance on FRET, Ryan Russell for critical reading of the manuscript, and NIH Grant P30 CA23100 for covering part of the cost for confocal imaging. This work was supported by grants from the NIH (to K.L.G.).

\section{References}

Avruch J, Praskova M, Ortiz-Vega S, Liu M, Zhang XF. 2006. Nore 1 and RASSF1 regulation of cell proliferation and of the MST1/2 kinases. Methods Enzymol 407: 290-310.

Badouel C, Gardano L, Amin N, Garg A, Rosenfeld R, Le Bihan T, McNeill H. 2009. The FERM-domain protein Expanded regulates Hippo pathway activity via direct interactions with the transcriptional activator Yorkie. Dev Cell 16: 411-420.

Baldwin C, Garnis C, Zhang L, Rosin MP, Lam WL. 2005. Multiple microalterations detected at high frequency in oral cancer. Cancer Res 65: 7561-7567.

Bratt A, Wilson WJ, Troyanovsky B, Aase K, Kessler R, Van Meir EG, Holmgren L. 2002. Angiomotin belongs to a novel protein family with conserved coiled-coil and PDZ binding domains. Gene 298: 69-77. 
Callus BA, Verhagen AM, Vaux DL. 2006. Association of mammalian sterile twenty kinases, Mst1 and Mst2, with hSalvador via C-terminal coiled-coil domains, leads to its stabilization and phosphorylation. FEBS J 273: 4264-4276.

Camargo FD, Gokhale S, Johnnidis JB, Fu D, Bell GW, Jaenisch R, Brummelkamp TR. 2007. YAP1 increases organ size and expands undifferentiated progenitor cells. Curr Biol 17: 2054-2060.

Chan EH, Nousiainen M, Chalamalasetty RB, Schafer A, Nigg EA, Sillje HH. 2005. The Ste20-like kinase Mst2 activates the human large tumor suppressor kinase Lats1. Oncogene 24: 2076-2086.

Chan SW, Lim CJ, Guo K, Ng CP, Lee I, Hunziker W, Zeng Q, Hong W. 2008. A role for TAZ in migration, invasion, and tumorigenesis of breast cancer cells. Cancer Res 68: 2592-2598.

Dong J, Feldmann G, Huang J, Wu S, Zhang N, Comerford SA, Gayyed MF, Anders RA, Maitra A, Pan D. 2007. Elucidation of a universal size-control mechanism in Drosophila and mammals. Cell 130: 1120-1133.

Ernkvist M, Aase K, Ukomadu C, Wohlschlegel J, Blackman R, Veitonmaki N, Bratt A, Dutta A, Holmgren L. 2006. p130angiomotin associates to actin and controls endothelial cell shape. FEBS I 273: 2000-2011.

Ernkvist M, Birot O, Sinha I, Veitonmaki N, Nystrom S, Aase K, Holmgren L. 2008. Differential roles of p80- and p130angiomotin in the switch between migration and stabilization of endothelial cells. Biochim Biophys Acta 1783:429_ 437.

Fernandez LA, Northcott PA, Dalton J, Fraga C, Ellison D, Angers S, Taylor MD, Kenney AM. 2009. YAP1 is amplified and up-regulated in hedgehog-associated medulloblastomas and mediates Sonic hedgehog-driven neural precursor proliferation. Genes Dev 23: 2729-2741.

Grzeschik NA, Parsons LM, Allott ML, Harvey KF, Richardson HE. 2010. Lgl, aPKC, and Crumbs regulate the Salvador/ Warts/Hippo pathway through two distinct mechanisms. Curr Biol 20: 573-581.

Hao Y, Chun A, Cheung K, Rashidi B, Yang X. 2008. Tumor suppressor LATS1 is a negative regulator of oncogene YAP. I Biol Chem 283: 5496-5509.

Harvey KF, Pfleger CM, Hariharan IK. 2003. The Drosophila Mst ortholog, hippo, restricts growth and cell proliferation and promotes apoptosis. Cell 114: 457-467.

Hergovich A, Schmitz D, Hemmings BA. 2006. The human tumour suppressor LATS1 is activated by human MOB1 at the membrane. Biochem Biophys Res Commun 345: 50-58.

Ho LL, Wei X, Shimizu T, Lai ZC. 2009. Mob as tumor suppressor is activated at the cell membrane to control tissue growth and organ size in Drosophila. Dev Biol 337: 274-283.

Hogan C, Dupre-Crochet S, Norman M, Kajita M, Zimmermann C, Pelling AE, Piddini E, Baena-Lopez LA, Vincent JP, Itoh Y, et al. 2009. Characterization of the interface between normal and transformed epithelial cells. Nat Cell Biol 11: 460-467.

Huang J, Wu S, Barrera J, Matthews K, Pan D. 2005. The Hippo signaling pathway coordinately regulates cell proliferation and apoptosis by inactivating Yorkie, the Drosophila homo$\log$ of YAP. Cell 122: 421-434.

Jia J, Zhang W, Wang B, Trinko R, Jiang J. 2003. The Drosophila Ste20 family kinase dMST functions as a tumor suppressor by restricting cell proliferation and promoting apoptosis. Genes Dev 17: 2514-2519.

Kango-Singh M, Nolo R, Tao C, Verstreken P, Hiesinger PR, Bellen HJ, Halder G. 2002. Shar-pei mediates cell proliferation arrest during imaginal disc growth in Drosophila. Development 129: 5719-5730.
Lai ZC, Wei X, Shimizu T, Ramos E, Rohrbaugh M, Nikolaidis N, Ho LL, Li Y. 2005. Control of cell proliferation and apoptosis by mob as tumor suppressor, mats. Cell 120: 675-685.

Ling C, Zheng Y, Yin F, Yu J, Huang J, Hong Y, Wu S, Pan D. 2010. The apical transmembrane protein Crumbs functions as a tumor suppressor that regulates Hippo signaling by binding to Expanded. Proc Natl Acad Sci 107: 1053210537.

Modena P, Lualdi E, Facchinetti F, Veltman J, Reid JF, Minardi S, Janssen I, Giangaspero F, Forni M, Finocchiaro G, et al. 2006. Identification of tumor-specific molecular signatures in intracranial ependymoma and association with clinical characteristics. J Clin Oncol 24: 5223-5233.

Nolo R, Morrison CM, Tao C, Zhang X, Halder G. 2006. The bantam microRNA is a target of the hippo tumor-suppressor pathway. Curr Biol 16: 1895-1904.

Oh H, Reddy BV, Irvine KD. 2009. Phosphorylation-independent repression of Yorkie in Fat-Hippo signaling. Dev Biol 335: $188-197$.

Oka T, Mazack V, Sudol M. 2008. Mst2 and Lats kinases regulate apoptotic function of Yes kinase-associated protein (YAP). J Biol Chem 283: 27534-27546.

Overholtzer M, Zhang J, Smolen GA, Muir B, Li W, Sgroi DC, Deng CX, Brugge JS, Haber DA. 2006. Transforming properties of YAP, a candidate oncogene on the chromosome 11q22 amplicon. Proc Natl Acad Sci 103: 12405-12410.

Pantalacci S, Tapon N, Leopold P. 2003. The Salvador partner Hippo promotes apoptosis and cell-cycle exit in Drosophila. Nat Cell Biol 5: 921-927.

Robinson BS, Huang J, Hong Y, Moberg KH. 2010. Crumbs regulates Salvador/Warts/Hippo signaling in Drosophila via the FERM-domain protein expanded. Curr Biol 20: 582-590.

Shimono A, Behringer RR. 2003. Angiomotin regulates visceral endoderm movements during mouse embryogenesis. Curr Biol 13: 613-617.

Snijders AM, Schmidt BL, Fridlyand J, Dekker N, Pinkel D, Jordan RC, Albertson DG. 2005. Rare amplicons implicate frequent deregulation of cell fate specification pathways in oral squamous cell carcinoma. Oncogene 24: 4232-4242.

Song H, Mak KK, Topol L, Yun K, Hu J, Garrett L, Chen Y, Park O, Chang J, Simpson RM, et al. 2010. Mammalian Mst1 and Mst2 kinases play essential roles in organ size control and tumor suppression. Proc Natl Acad Sci 107: 1431-1436.

Steinhardt AA, Gayyed MF, Klein AP, Dong J, Maitra A, Pan D, Montgomery EA, Anders RA. 2008. Expression of Yes-associated protein in common solid tumors. Hum Pathol 39: 1582-1589.

Tapon N, Harvey KF, Bell DW, Wahrer DC, Schiripo TA, Haber DA, Hariharan IK. 2002. salvador promotes both cell cycle exit and apoptosis in Drosophila and is mutated in human cancer cell lines. Cell 110: 467-478.

Thompson BJ, Cohen SM. 2006. The Hippo pathway regulates the bantam microRNA to control cell proliferation and apoptosis in Drosophila. Cell 126: 767-774.

Troyanovsky B, Levchenko T, Mansson G, Matvijenko O, Holmgren L. 2001. Angiomotin: An angiostatin binding protein that regulates endothelial cell migration and tube formation. J Cell Biol 152: 1247-1254.

Udan RS, Kango-Singh M, Nolo R, Tao C, Halder G. 2003. Hippo promotes proliferation arrest and apoptosis in the Salvador/ Warts pathway. Nat Cell Biol 5: 914-920.

Wells CD, Fawcett JP, Traweger A, Yamanaka Y, Goudreault M, Elder K, Kulkarni S, Gish G, Virag C, Lim C, et al. 2006. A Rich1/Amot complex regulates the Cdc42 GTPase and apical-polarity proteins in epithelial cells. Cell 125: 535-548.

Wu S, Huang J, Dong J, Pan D. 2003. hippo encodes a Ste-20 family protein kinase that restricts cell proliferation and 
promotes apoptosis in conjunction with salvador and warts. Cell 114: 445-456.

Xu MZ, Yao TJ, Lee NP, Ng IO, Chan YT, Zender L, Lowe SW, Poon RT, Luk JM. 2009. Yes-associated protein is an independent prognostic marker in hepatocellular carcinoma. Cancer 115: 4576-4585.

Zender L, Spector MS, Xue W, Flemming P, Cordon-Cardo C, Silke J, Fan ST, Luk JM, Wigler M, Hannon GJ, et al. 2006. Identification and validation of oncogenes in liver cancer using an integrative oncogenomic approach. Cell 125: 1253-1267.

Zhang J, Smolen GA, Haber DA. 2008. Negative regulation of YAP by LATS1 underscores evolutionary conservation of the Drosophila Hippo pathway. Cancer Res 68: 2789-2794.

Zhang J, Ji JY, Yu M, Overholtzer M, Smolen GA, Wang R, Brugge JS, Dyson NJ, Haber DA. 2009. YAP-dependent induction of amphiregulin identifies a non-cell-autonomous component of the Hippo pathway. Nat Cell Biol 11: 1444-1450.

Zhang X, Milton CC, Humbert PO, Harvey KF. 2009. Transcriptional output of the Salvador/warts/hippo pathway is controlled in distinct fashions in Drosophila melanogaster and mammalian cell lines. Cancer Res 69: 6033-6041.

Zhao B, Wei X, Li W, Udan RS, Yang Q, Kim J, Xie J, Ikenoue T, $\mathrm{Yu}$ J, Li L, et al. 2007. Inactivation of YAP oncoprotein by the Hippo pathway is involved in cell contact inhibition and tissue growth control. Genes Dev 21: 2747-2761.

Zhao B, Ye X, Yu J, Li L, Li W, Li S, Lin JD, Wang CY, Chinnaiyan AM, Lai ZC, et al. 2008. TEAD mediates YAP-dependent gene induction and growth control. Genes Dev 22: 1962-1971.

Zhao B, Kim J, Ye X, Lai ZC, Guan KL. 2009. Both TEAD-binding and WW domains are required for the growth stimulation and oncogenic transformation activity of yes-associated protein. Cancer Res 69: 1089-1098.

Zhao B, Li L, Lei Q, Guan KL. 2010a. The Hippo-YAP pathway in organ size control and tumorigenesis: An updated version. Genes Dev 24: 862-874.

Zhao B, Li L, Tumaneng K, Wang CY, Guan KL. 2010b. A coordinated phosphorylation by Lats and CK1 regulates YAP stability through SCF( $\beta-\mathrm{TRCP})$. Genes Dev 24: 72-85. 


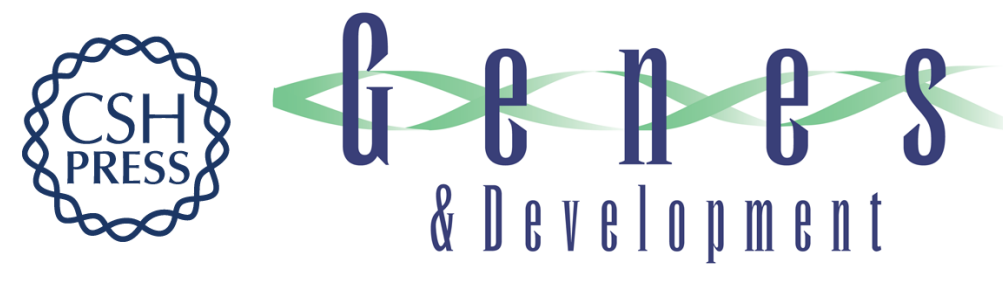

\section{Angiomotin is a novel Hippo pathway component that inhibits YAP oncoprotein}

Bin Zhao, Li Li, Qing Lu, et al.

Genes Dev. 2011, 25:

Access the most recent version at doi:10.1101/gad.2000111

Supplemental http://genesdev.cshlp.org/content/suppl/2010/12/28/25.1.51.DC1
Material

References This article cites 50 articles, 17 of which can be accessed free at: http://genesdev.cshlp.org/content/25/1/51.full.html\#ref-list-1

License

Email Alerting Receive free email alerts when new articles cite this article - sign up in the box at the top Service right corner of the article or click here.

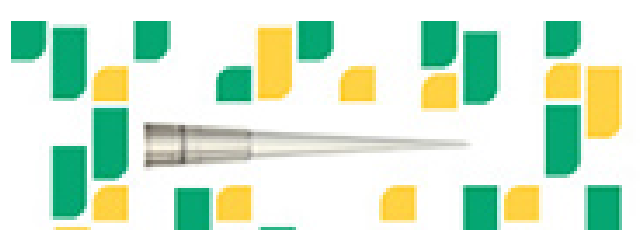

Focused on your science. 